\title{
Entanglement-induced decoherence and energy eigenstates
}

\author{
Wen-ge Wang, ${ }^{1,2,3, *}$ Jiangbin Gong, ${ }^{1,4}$ G. Casati, ${ }^{1,5,6}$ and Baowen $\mathrm{Li}^{1,4}$ \\ ${ }^{1}$ Department of Physics and Centre for Computational Science and Engineering, National University of Singapore, \\ Singapore 117542, Republic of Singapore \\ ${ }^{2}$ Department of Modern Physics, University of Science and Technology of China, Hefei 230026, China \\ ${ }^{3}$ Department of Physics, Southeast University, Nanjing 210096, China \\ ${ }^{4}$ NUS Graduate School for Integrative Sciences and Engineering, Singapore 117597, Republic of Singapore \\ ${ }^{5}$ Center for Nonlinear and Complex Systems, Università degli Studi dell'Insubria, Via Valleggio 11, 22100 Como, Italy \\ ${ }^{6}$ CNR-INFM and Istituto Nazionale di Fisica Nucleare, Sezione di Milano, Milan, Italy
}

(Received 23 August 2007; published 22 January 2008)

\begin{abstract}
Using recent results in the field of quantum chaos we derive explicit expressions for the time scale of decoherence induced by the system-environment entanglement. For a generic system-environment interaction and for a generic quantum chaotic system as environment, conditions are derived for energy eigenstates to be preferred states in the weak coupling regime. A simple model is introduced to numerically confirm our predictions. The results presented here may also help with understanding the dynamics of quantum entanglement generation in chaotic quantum systems.
\end{abstract}

DOI: 10.1103/PhysRevA.77.012108

PACS number(s): 03.65.Yz, 03.65.Ta, 03.67.Mn, 05.45.Mt

\section{INTRODUCTION}

Real physical systems are never isolated from the surrounding world and, as a consequence, nonclassical correlations (entanglement) are established between the system and the environment. This process, which leads to decoherence, has a fundamental interest since it contributes to the understanding of the emergence of classicality in a world governed by the laws of quantum mechanics [1].

Remarkably, different states may decohere at drastically different rates, and a small fraction of them may be particularly stable under entangling interaction with the environment $[1,2]$. Such states are "preferred states" of the system under the influence of the environment. (They are also called "pointer states," a name given for the states of pointers of measurement apparatus in the study of the measurement problem [1].) This concept is important in understanding what states will naturally emerge from a quantum system subject to decoherence.

As discussed by Paz and Zurek in an interesting paper [3], depending on the type and strength of the systemenvironment interaction, different preferred states may arise during the decoherence process. In particular they consider the case of an adiabatic environment modeled by a quantum scalar field and interacting weakly with a system through a given type of coupling. In such situation they show that energy eigenstates are good preferred states and hence are the natural representation of the quantum system. Notice that the adiabatic environment does not change the population of energy eigenstates of the system, implying infinite relaxation time.

In realistic situations the environment is often nonadiabatic, with finite relaxation time. In this case, the relation between the relaxation time and the decoherence time is crucial and, typically, only when the former is much longer than

\footnotetext{
*wgwang@ustc.edu.cn
}

the latter, energy eigenstates can be good preferred states. While a rough estimate of the relaxation time can be obtained via Fermi's golden rule, for the decoherence time the situation is more complex. Indeed in the case of a generic type of coupling and generic environment, it is hardly possible to obtain estimates by employing the master-equation approach used in [3].

In this paper we propose an alternative approach which takes advantage of recent progress in the field of quantum chaos: namely random matrix theory and the so-called fidelity [4-8] which is a measure of the stability of the quantum motion under system perturbations. Using this approach we can estimate - via the fidelity decay of the environment- the decoherence time of the system for weak, generic systemenvironment couplings and for a broad class of environments. In particular, we need not restrict ourselves to the Markovian regime (in contrast to master-equation approach) where the bath correlation decay is faster than decoherence. Moreover, as an important result of ours, by modeling the environment by a chaotic quantum system, we determine a critical border in the coupling strength below which energy eigenstates are shown to be preferred states, while above this border, the relaxation time and decoherence time have the same scaling with the coupling strength and therefore no definite statements can be made. We also present numerical results which confirm the above picture.

\section{GENERAL THEORY}

Let us consider a quantum system $S$ with a discrete spectrum, weakly coupled to a second quantum system $\mathcal{E}$ as its environment. The total Hamiltonian is

$$
H=H_{S}+\epsilon H_{I}+H_{\mathcal{E}},
$$

where $H_{S}$ and $H_{\mathcal{E}}$ are the Hamiltonians of $S$ and $\mathcal{E}$, respectively, and $\epsilon H_{I}$ is the weak interaction Hamiltonian $(\epsilon \ll 1)$. The time evolution of the whole system is given by $\left|\Psi_{S \mathcal{E}}(t)\right\rangle=e^{-i H t / \hbar}\left|\Psi_{S \mathcal{E}}(0)\right\rangle$. The initial state is set as a product 
state $\left|\Psi_{S \mathcal{E}}(0)\right\rangle=\left|\psi_{S}(0)\right\rangle\left|\phi_{\mathcal{E}}(0)\right\rangle$. The reduced density matrix $\left\langle\alpha\left|\rho^{r e}(t)\right| \beta\right\rangle=\left\langle\alpha\left|\operatorname{Tr}_{\mathcal{E}} \rho(t)\right| \beta\right\rangle$ is obtained by tracing over the environment.

Consider first the case with initial state $\left|\psi_{S}(0)\right\rangle=|\alpha\rangle$, where $|\alpha\rangle$ denotes an energy eigenstate of $H_{S}$ with eigenenergy $E_{\alpha}$. We define the projection operators $|\alpha\rangle\langle\alpha| \otimes 1_{\mathcal{E}}$ and $\mathcal{P}_{\bar{\alpha}} \equiv \Sigma_{\beta \neq \alpha}|\beta\rangle\langle\beta| \otimes 1_{\mathcal{E}}$ where $1_{\mathcal{E}}$ is the identity operator for the environment degrees of freedom. The whole Hilbert space can then be decomposed into two orthogonal subspaces, leading to

$$
\left|\Psi_{S \mathcal{E}}(t)\right\rangle=e^{-i E_{\alpha} t / \hbar}|\alpha\rangle\left|\phi_{\alpha}^{\mathcal{E}}(t)\right\rangle+\epsilon\left|\chi_{\bar{\alpha}}(t)\right\rangle,
$$

where $\epsilon\left|\chi_{\bar{\alpha}}(t)\right\rangle \equiv \mathcal{P}_{\bar{\alpha}}\left|\Psi_{S \mathcal{E}}(t)\right\rangle$. The small parameter $\epsilon$ is introduced to account for the fact that, in case of weak coupling, the second term in Eq. (2) remains small inside some initial time interval (see below). The normalization of $\left|\Psi_{S \mathcal{E}}(t)\right\rangle$ in Eq. (2) is unity to the first order in $\epsilon$.

A simple derivation shows that the evolution of the two terms in Eq. (2) is given by the coupled equations,

$$
\begin{gathered}
i \hbar \frac{d}{d t}\left|\phi_{\alpha}^{\mathcal{E}}(t)\right\rangle=H_{\mathcal{E} \alpha}^{\mathrm{eff}}\left|\phi_{\alpha}^{\mathcal{E}}(t)\right\rangle+\epsilon^{2} e^{i E_{\alpha} t / \hbar}\left\langle\alpha\left|H_{I}\right| \chi_{\bar{\alpha}}(t)\right\rangle, \\
i \hbar \frac{d}{d t}\left|\eta_{\bar{\alpha}}(t)\right\rangle=\exp \left(-\frac{i}{\hbar}\left(E_{\alpha}-H_{\bar{\alpha}}\right) t\right) \mathcal{P}_{\bar{\alpha}} H_{I}|\alpha\rangle\left|\phi_{\alpha}^{\mathcal{E}}(t)\right\rangle,
\end{gathered}
$$

where $H_{\mathcal{E} \alpha}^{\text {eff }} \equiv \epsilon H_{I \alpha}+H_{\mathcal{E}}, \quad\left|\eta_{\bar{\alpha}}(t)\right\rangle \equiv \exp \left(i H_{\bar{\alpha}} t / \hbar\right)\left|\chi_{\bar{\alpha}}(t)\right\rangle, \quad H_{I \alpha}$ $\equiv\left\langle\alpha\left|H_{I}\right| \alpha\right\rangle$, and $H_{\bar{\alpha}} \equiv \mathcal{P}_{\bar{\alpha}} H \mathcal{P}_{\bar{\alpha}}$.

It is evident from Eq. (2) that $\epsilon^{2}\left\langle\chi_{\bar{\alpha}} \mid \chi_{\bar{\alpha}}\right\rangle$ gives the population that has leaked to the subspace associated with $\mathcal{P}_{\bar{\alpha}}$. In the case of weak coupling, this population leakage is initially very small. More precisely we have that $\epsilon^{2}\left\langle\chi_{\bar{\alpha}} \mid \chi_{\bar{\alpha}}\right\rangle \ll 1$ up to times $t \ll \tau_{E}$, where $\tau_{E}$ is the relaxation time of the system. Then the second term in Eq. (3) can be safely neglected and, as a result, the environment is in the state $\left|\phi_{\alpha}^{\mathcal{E}}(t)\right\rangle$ $\approx e^{-i t H_{\mathcal{E} \alpha}^{\mathrm{eff}} / \hbar}\left|\phi_{\mathcal{E}}(0)\right\rangle$ while the system remains in the eigenstate $|\alpha\rangle$ with a definite phase evolution.

Consider now as the initial state a superposition of energy eigenstates $\left|\psi_{S}(0)\right\rangle=\Sigma_{\alpha} C_{\alpha}|\alpha\rangle$. As in Eq. (2) we can write

$$
\left|\Psi_{S \mathcal{E}}(t)\right\rangle=\sum_{\alpha} e^{-i E_{\alpha} t / \hbar} C_{\alpha}|\alpha\rangle\left|\phi_{\alpha}^{\mathcal{E}}(t)\right\rangle+\epsilon|\chi(t)\rangle
$$

where the term $|\chi(t)\rangle=\Sigma_{\alpha} C_{\alpha}\left|\chi_{\bar{\alpha}}(t)\right\rangle$ contains now contributions of transitions between different energy eigenstates. Note that the first term on the right-hand side of Eq. (5) may be highly entangled even when $\epsilon|\chi(t)\rangle$ is small. For $t \ll \tau_{E}$, the term $\epsilon|\chi(t)\rangle$ in Eq. (5) can be neglected and, by tracing out the environment, the reduced density matrix of the system can be written as

$$
\rho_{\alpha \beta}^{r e}=\left\langle\alpha\left|\operatorname{Tr}_{\mathcal{E}} \rho(t)\right| \beta\right\rangle \simeq e^{-i\left(E_{\alpha}-E_{\beta}\right) t / \hbar} C_{\alpha} C_{\beta}^{*} f_{\beta \alpha}(t),
$$

where $f_{\beta \alpha}(t) \equiv\left\langle\phi_{\beta}^{\mathcal{E}}(t) \mid \phi_{\alpha}^{\mathcal{E}}(t)\right\rangle$ satisfies

$$
f_{\beta \alpha}(t) \approx\left\langle\phi_{\mathcal{E}}(0)\left|e^{i t\left(H_{\mathcal{E} \alpha}^{\mathrm{eff}}+\epsilon V\right) / \hbar} e^{-i t H_{\mathcal{E} \alpha}^{\mathrm{eff}} / \hbar}\right| \phi_{\mathcal{E}}(0)\right\rangle,
$$

with

$$
V \equiv H_{I \beta}-H_{I \alpha}=\left\langle\beta\left|H_{I}\right| \beta\right\rangle-\left\langle\alpha\left|H_{I}\right| \alpha\right\rangle .
$$

Significantly, the quantity $f_{\beta \alpha}(t)$ is simply the "fidelity amplitude" of the environment associated with the two slightly different Hamiltonians $H_{\mathcal{E} \alpha}^{\text {eff }}$ and $\left(H_{\mathcal{E} \alpha}^{\text {eff }}+\epsilon V\right)$. For nonconstant $V$, this fidelity amplitude usually decays with time for $\alpha$ $\neq \beta$, then $\rho_{\alpha \beta}^{r e}$ also decays and therefore decoherence sets in. In the case of constant $V$ in Eq. (8), Eq. (7) gives $\left|f_{\beta \alpha}(t)\right|$ $\approx 1$, hence, there is no decoherence induced by the environment in the first-order perturbation theory discussed above. Notice that Eqs. (6) and (7) become exact in the particular case in which the coupling $H_{I}$ commutes with $H_{S}$. This case has been considered in [9].

It turns out, from the above considerations, that for weak, but generic coupling, energy eigenstates of $H_{S}$ play a special role in the sense that, only for energy superposition states (not single eigenstates), the decoherence process is associated with the instability of the environment under perturbation (fidelity decay).

\section{A MODEL WITH THE ENVIRONMENT MODELED BY A QUANTUM CHAOTIC SYSTEM}

Let us now turn to an explicit estimate of the decoherence time of the system. To this end we can directly apply recent results on fidelity decay [4-7] which, as shown below, allows us to estimate the decoherence time for a generic type of system-environment interaction and for a broad class of environments. This contrasts the situation of the masterequation approach with which only particular types of interaction and environment have been treated [3] while extension to more general situations is mathematically difficult.

Let us assume that the environment is modeled by a quantum chaotic system [10]. (Analogous strategy can be applied when the environment has a regular or mixed-type phase space structure.) Fidelity decay in such systems has been studied via semiclassical methods as well as with random matrix theory, both giving consistent results. Specifically, it turns out that for initial states chosen randomly, the fidelity has typically a Gaussian decay below a perturbative border $\epsilon_{p}$ and an exponential decay above this border [4-6]. If we model the environment $H_{\mathcal{E}}$ by a single matrix of dimension $N$ taken from the so-called Gaussian orthogonal ensemble (GOE) [11], then the border $\epsilon_{p}$ can be explicitly estimated and is given by [5]

$$
2 \pi \epsilon_{p} \overline{V_{n d}^{2}} \sim \sigma_{v} \Delta
$$

where $\overline{V_{n d}^{2}}$ is the average of $\left|\left\langle n|V| n^{\prime}\right\rangle\right|^{2}$ with $n \neq n^{\prime}$ and $V$ given by Eq. (8). Here $|n\rangle$ denotes the eigenstates of $H_{\mathcal{E} \alpha}^{\text {eff }}, \Delta$ is the mean level spacing of $H_{\mathcal{E} \alpha}^{\mathrm{eff}}$, and $\sigma_{v}^{2}$ is the variance of $\langle n|V| n\rangle$.

Below the perturbative border, $\epsilon<\epsilon_{p}$, the fidelity amplitude decays as [5]

$$
\left|f_{\beta \alpha}(t)\right| \simeq e^{-\epsilon^{2} \sigma_{v}^{2} t^{2} / 2 \hbar^{2}} .
$$

Then, the decoherence time $\tau_{d}$, characterizing the decay of off-diagonal matrix elements [see Eq. (6)] is given by 


$$
\tau_{d} \simeq \sqrt{2} \hbar /\left(\epsilon \sigma_{v}\right) \propto \epsilon^{-1}, \quad \epsilon<\epsilon_{p} .
$$

Notice that this dependence of $\tau_{d}$ on $\epsilon$ coincides with the one derived in Ref. [3], even though in our case we do not assume an adiabatic environment. For $\epsilon>\epsilon_{p},\left|f_{\beta \alpha}(t)\right|$ has an exponential decay [4],

$$
\left|f_{\beta \alpha}(t)\right| \sim e^{-\Gamma t / 2 \hbar} \quad \text { with } \Gamma=2 \pi \epsilon^{2} \overline{V_{n d}^{2}} / \Delta,
$$

and

$$
\tau_{d} \simeq \hbar \Delta /\left(\pi \epsilon^{2} \overline{V_{n d}^{2}}\right) \propto \epsilon^{-2}, \quad \epsilon>\epsilon_{p} .
$$

We stress that, like Eq. (6), also Eqs. (11) and (13) are valid only if the time scale under consideration is much less than $\tau_{E}$.

The next issue is if, and under what conditions, $\tau_{E}$ is sufficiently large so that significant decoherence may occur for $t \ll \tau_{E}$. If this is the case, then energy eigenstates are much more robust than their superposition states. Let $\left|\mu_{\mathcal{E}}\right\rangle$ be one eigenstate of $H_{\mathcal{E}}$ and $\left\langle H_{I, n d}^{2}\right\rangle$ be the mean square of the nondiagonal matrix elements $\left\langle\alpha^{\prime}\left|\left\langle\mu_{\mathcal{E}}^{\prime}\left|H_{I}\right| \mu_{\mathcal{E}}\right\rangle\right| \alpha\right\rangle \quad\left(\alpha \neq \alpha^{\prime}\right)$. One may estimate $\tau_{E}$ by using Fermi's golden rule, i.e.,

$$
\tau_{E} \simeq 1 / R \propto \epsilon^{-2}, \quad \text { where } R=2 \pi \epsilon^{2} \rho\left\langle H_{I, n d}^{2}\right\rangle / \hbar
$$

is the transition rate in Fermi's golden rule with the on-shell density-of-states $\rho$ approximated by the average density of all possible final states for the whole system [12].

Therefore, below the perturbative border $\left(\epsilon<\epsilon_{p}\right)$ the decoherence time $\tau_{d}$ and the relaxation time $\tau_{E}$ scale as $\epsilon^{-1}$ and $\epsilon^{-2}$, respectively. It follows that for small enough $\epsilon$, we have $\tau_{d} \ll \tau_{E}$ and hence energy eigenstates are good preferred states regardless of the form of the coupling. On the other hand, above the perturbative border, both $\tau_{d}$ in Eq. (13) and $\tau_{E}$ in Eq. (14) scale with $\epsilon^{-2}$. In particular, in cases of very small $\epsilon_{p}$, the two time scales can be comparable [13] even at weak perturbation and hence energy eigenstates may not be preferred states.

We will now introduce a simple dimensionless model which will also allow an explicit numerical evaluation of the different time scales. Let $S$ be a qubit system with Hamiltonian $H_{S}=\Sigma_{\alpha} E_{\alpha}|\alpha\rangle\langle\alpha|, \alpha=1,2$ and $E_{2}-E_{1}=1$. The environment is modeled by a $N$-dimensional matrix in the GOE and the interaction $H_{I}$ is taken in the form of a random matrix as well. Specifically, in a given arbitrary basis $|k\rangle$, the matrix elements $\left\langle k\left|H_{\mathcal{E}}\right| k^{\prime}\right\rangle$ and $\left\langle\alpha k\left|H_{I}\right| \alpha^{\prime} k^{\prime}\right\rangle$ are real random numbers distributed according to a Gaussian with unit variance. Moreover, we set the Planck constant $\hbar=1$.

We have numerically integrated the time-dependent Schrödinger equation for this model thus obtaining the matrix elements of the reduced density matrix $\rho^{r e}$. In Fig. 1, we show the numerical results for parameters $N=200$ and $\epsilon=5$ $\times 10^{-4}$. The perturbative border can be numerically computed from Eq. (9) and it is found to be $\epsilon_{p} \sim 0.04$. It is clearly seen that for weak coupling the decay of $\rho_{12}^{r e}$ is well predicted by the Gaussian decay in Eq. (10). By contrast, the change in the diagonal matrix elements of the reduced density is negligible, as shown by the upper thin curve in Fig. 1.

Figure 2 is drawn for the same parameters of Fig. 1 but

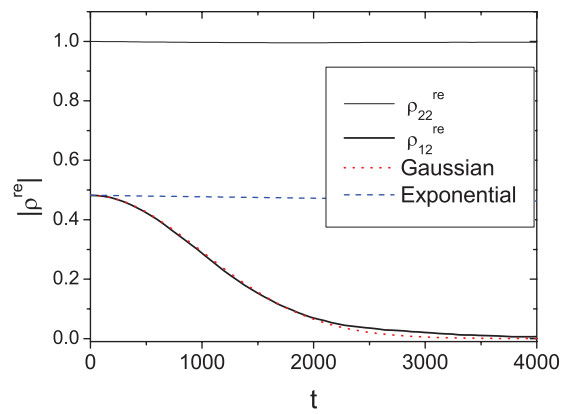

FIG. 1. (Color online) Time dependence of the elements of the reduced density matrix of the qubit system $S$. Here $N=200$ and the coupling strength $\epsilon=5 \times 10^{-4}$ is much smaller than the perturbative border $\epsilon_{p} \sim 0.04$ numerically computed from Eq. (9). The upper thin solid curve gives the diagonal matrix element $\rho_{22}^{r e}(t)$ for an initial state with $\left|\psi_{S}(0)\right\rangle=|2\rangle$. The lower thick solid curve gives the offdiagonal matrix element $\rho_{12}^{r e}(t)$ for an initial state which is a product state in which the system $S$ is in an energy superposition state while the environment is in a randomly chosen state. The dotted and dashed curves give the theoretical Gaussian decay equation (10) and the exponential decay equation (12), respectively.

for a larger coupling strength, comparable to the perturbative border. One notices deviations from the Gaussian decay and also an appreciable population change (upper thin curve). Interestingly, we found that deviations from the Gaussian decay always goes with an appreciable population change. Indeed if, for example, we deliberately weaken those offdiagonal coupling terms (diagonal coupling terms not touched) that are responsible for the population decay, then the Gaussian decay can be recovered while the population decay becomes negligible.

Finally, if we further increase the coupling strength above the perturbative border, then the exponential decay of the off-diagonal matrix elements [Eq. (12)] is indeed observed (not shown here).

We have carefully studied the scaling behavior of $\tau_{E}$ and $\tau_{d}$ as functions of the coupling strength $\epsilon$ and the results are shown in Fig. 3. Numerically $\tau_{d}$ is defined as the time scale over which $\left|\rho_{12}^{r e}\right|$ decays by a factor of $1 / e$, and $\tau_{E}$ is defined as the reciprocal of the slope of $\rho_{22}^{r e}(t)$ in the initial interval of

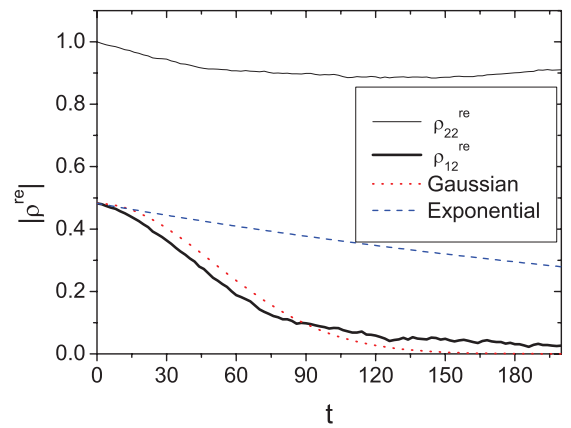

FIG. 2. (Color online) Same as in Fig. 1 but for $\epsilon=0.01$ which is still below but close to the perturbation border. It is seen that the numerically computed $\left|\rho_{12}^{r e}\right|$ begin to deviate from the predicted Gaussian decay. Moreover the population decay (thin solid curve) becomes appreciable. 


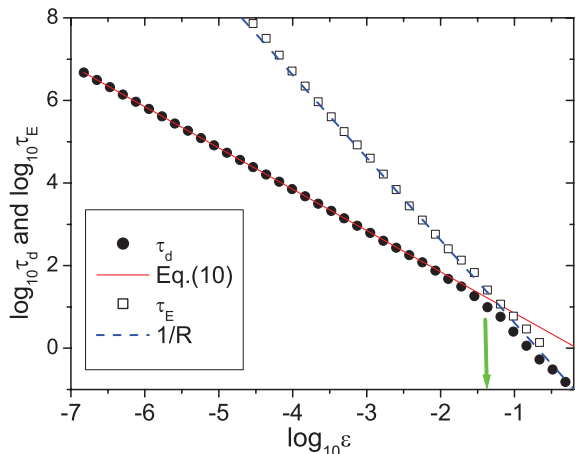

FIG. 3. (Color online) Dependence of times $\tau_{d}$ and $\tau_{E}$ on the coupling strength $\epsilon$. Full circles and empty squares represent the numerically computed $\tau_{d}$ and $\tau_{E}$, respectively. The solid line is given by the theoretical expression (11) and the dashed line is given by Eq. (14). Notice the good agreement between numerical data and theoretical predictions. The arrow indicates the perturbative border $\epsilon_{p}$ which clearly separates the two different scaling behaviors of $\tau_{d}$.

time in which Fermi's golden rule is valid. It is seen that numerical data nicely agree with analytical predictions. In particular, one can distinguish between the two scaling behaviors of $\tau_{d}\left(\epsilon^{-1}\right.$ and $\left.\epsilon^{-2}\right)$ separated by the perturbative border $\epsilon_{p}$. Below this border, $\tau_{d} \ll \tau_{E}$, implying that energy eigenstates are much more stable than energy superposition states.

\section{DISCUSSIONS AND CONCLUSIONS}

Entanglement-induced decoherence within a closed total system is also referred to as "intrinsic decoherence" [14].
The above analysis directly indicates the existence of preferred states of intrinsic decoherence. As such, the dynamics of entanglement generation between two subsystems depends strongly on the coherence properties of the initial state. This is of interest to studies of entanglement generation in classically chaotic systems [15]. Further, our results might also shed light on the dynamics of quantum thermalization processes within a closed system [16], where energy eigenstates also play a special role.

In summary, for weak but generic coupling between two quantum subsystems, energy eigenstates are shown to play a special role in the entanglement-induced decoherence process. The quality of the energy eigenstates as preferred states is also analyzed in terms of a simple dynamical model which allows for numerical analysis.

\section{ACKNOWLEDGMENTS}

The authors are very grateful to F. Haake for valuable discussions and suggestions. This work is supported in part by an Academic Research Fund of NUS and the TYIA of DSTA, Singapore under Contract No. POD0410553. One of the authors (W.W.) is partially supported by Natural Science Foundation of China Grants No. 10275011 and No. 10775123. One of the authors (J.G.) is supported by the start-up funding and the NUS "YIA" funding (WBS Grant No. R-144-000-195-123). One of the authors (G.C.) is funded by the MIUR-PRIN 2005 "Quantum computation with trapped particle arrays, neutral and charged."
[1] W. H. Zurek, Phys. Rev. D 24, 1516 (1981); W. H. Zurek, S. Habib, and J. P. Paz, Phys. Rev. Lett. 70, 1187 (1993).

[2] D. Braun, F. Haake, and W. T. Strunz, Phys. Rev. Lett. 86, 2913 (2001); L. Diósi and C. Kiefer, ibid. 85, 3552 (2000); J. Eisert, ibid. 92, 210401 (2004); H. M. Wiseman and J. A. Vaccaro, Phys. Rev. A 65, 043606 (2002).

[3] J. P. Paz and W. H. Zurek, Phys. Rev. Lett. 82, 5181 (1999).

[4] R. A. Jalabert and H. M. Pastawski, Phys. Rev. Lett. 86, 2490 (2001); Ph. Jacquod, P. G. Silvestrov, and C. W. J. Beenakker, Phys. Rev. E 64, 055203(R) (2001); T. Prosen and M. Žnidarič, J. Phys. A 35, 1455 (2002); F. M. Cucchietti, C. H. Lewenkopf, E. R. Mucciolo, H. M. Pastawski, and R. O. Vallejos, Phys. Rev. E 65, 046209 (2002).

[5] N. R. Cerruti and S. Tomsovic, J. Phys. A 36, 3451 (2003); Phys. Rev. Lett. 88, 054103 (2002).

[6] W.-G. Wang and B. Li, Phys. Rev. E 66, 056208 (2002); W.-G. Wang, G. Casati, and B. Li, ibid. 69, 025201(R) (2004); W. Wang, G. Casati, B. Li, and T. Prosen, ibid. 71, 037202 (2005); W.-G. Wang and B. Li, ibid. 71, 066203 (2005).

[7] T. Gorin, T. Prosen, T. H. Seligman, and M. Žnidarič, Phys. Rep. 435, 33 (2006).

[8] F. M. Cucchietti, D. A. R. Dalvit, J. P. Paz, and W. H. Zurek, Phys. Rev. Lett. 91, 210403 (2003); F. M. Cucchietti, J. P. Paz, and W. H. Zurek, Phys. Rev. A 72, 052113 (2005).

[9] T. Gorin, T. Prosen, T. H. Seligman, and W. T. Strunz, Phys. Rev. A 70, 042105 (2004); H. T. Quan, Z. Song, X. F. Liu, P.
Zanardi, and C. P. Sun, Phys. Rev. Lett. 96, 140604 (2006).

[10] M. Srednicki, Phys. Rev. E 50, 888 (1994).

[11] Decoherence effects with environments modeled by random matrices are recently considered in C. Pineda and T. H. Seligman, Phys. Rev. A 75, 012106 (2007).

[12] The same scaling with $\epsilon$ is derived in a more rigorous treatment for $M$-level quantum systems interacting with reservoirs in the thermodynamic limit in M. Merkli, I. M. Sigal, and G. P. Berman, Phys. Rev. Lett. 98, 130401 (2007). We also remark that the decoherence time given in that paper also scales as $\epsilon^{-2}$, where the initial state of the environment is assumed to be an equilibrium state and the off-diagonal elements of reduced density matrix are studied with respect to their equilibrium values.

[13] Similar to the well-known $T_{2}-T_{1}$ relation, the relaxation time scale $\tau_{E}$ sets an upper bound to $\tau_{d}$.

[14] J. Gong and P. Brumer, Phys. Rev. A 68, 022101 (2003).

[15] J. N. Bandyopadhyay and A. Lakshminarayan, Phys. Rev. Lett. 89, 060402 (2002); Phys. Rev. E 69, 016201 (2004); H. Fujisaki, T. Miyadera, and A. Tanaka, ibid. 67, 066201 (2003); R. Demkowicz-Dobrzański and M. Kuś, ibid. 70, 066216 (2004); K. Furuya, M. C. Nemes, and G. Q. Pellegrino, Phys. Rev. Lett. 80, 5524 (1998).

[16] S. Popescu, A. J. Short, and A. Winter, Nat. Phys. 2, 754 (2006); S. Lloyd, ibid. 2, 727 (2006); S. Goldstein, J. L. Lebowitz, R. Tumulka, and N. Zanghi, Phys. Rev. Lett. 96, 050403 (2006). 